\title{
Tunneled central venous catheterization, a viable option for long-term venous access in pediatric burn patients
}

\author{
Clayton Wagner MS, Andrea Hess BS, Jose Olascoaga MS, \\ Nicole Van Spronsen BS, John Griswold MD
}

\begin{abstract}
Introduction: Pediatric patients with severe burns often require long-term venous access over the course of their recovery. The need for long-term venous access in these critically ill patients often necessitates the placement of a central venous catheter (CVC). Many techniques exist for the establishment of a CVC in pediatric burn patients, and each technique poses its own set of inherent risks. No studies to date have clearly delineated the risk associated with tunneled central venous catheterization in the pediatric burn patient population. The primary aim of this study was to evaluate the use of tunneled CVCs in pediatric burn patients at the University Medical Center Hospital in Lubbock, Texas.

Methods: To evaluate this method of central venous catheterization, we retrospectively reviewed the charts of pediatric burn patients who received a tunneled CVC to determine the incidence of specific complications associated with this catheterization technique. We present our findings here in a case series format.

Results: Our initial search of patient charts yielded 86 potential candidates for inclusion in the study. After reviewing each chart, 26 pediatric patients were found to have received a CVC. Of these 26 patients, five met all of the inclusion criteria of our study. In these five patients, eight tunneled CVCs were placed. The average age of the patients in this series at the time of their respective burn injuries was 3.9 years old. Mean percent TBSA involvement was $38 \%$ with an average length of stay totaling 64.6 days. The average dwell time of the tunneled CVCs in this series was 28 days, and our analysis of the data revealed one tunneled catheterrelated infection and one hemodynamic complication.

Conclusions: Overall, our data show that placement of long-term tunneled CVCs in pediatric burn patients appears to be a relatively safe practice. However, our small sample size warrants more investigation into this topic.
\end{abstract}

Keywords: pediatrics, burns, central vein catheters

\section{BACKGROUND}

In response to severe burn injury, cells of the innate immune system migrate to affected tissues and

Corresponding author: Clayton Wagner

Contact Information: Clayton.wagner@ttuhsc.edu

DOI: $10.12746 /$ swrccc.v8i33.629 secrete pro-inflammatory mediators. If not managed, these molecules can cause major compartmental fluid shifts, hypovolemic shock, multiple organ dysfunction syndrome (MODS), and even death in burn patients. ${ }^{1}$ In this patient population, central venous access is often necessary for the administration of fluids, medications, and blood products. Placement of a central line is a routine procedure, but central venous access can be more complicated in certain patient populations. Burn and pediatric patients represent two of 
these populations due to decreased availability of anatomical sites for line placement and small vessel size, respectively. In pediatric burn patients, the difficulty associated with acquisition of central venous access can be greater than the challenge associated with this procedure in adult burn or pediatric patients combined.

Typically, central venous catheterization is required only in pediatric burn patients who are very ill; the extent of injury in these patients sometimes necessitates long-term venous access. Central venous catheters (CVCs) without subcutaneous tunnel and peripherally inserted central catheters (PICCs) are both universally accepted options for venous access in this patient population since these lines are easy to place, inexpensive, and have better passive flowrates. Exposure to anesthesia, which is often required for the placement of tunneled CVCs in burn patients, is often considered an unnecessary risk since non-tunneled CVCs and PICCs can be placed at the bedside. One of the primary drawbacks of CVCs without subcutaneous tunnel is the shorter dwell time of these lines. The Michigan Appropriateness Guide for Intravenous Catheters (MAGIC) guidelines suggest a 14-day dwell time for non-tunneled CVCs. ${ }^{2}$ The short dwell time of these lines often necessitates several line changes over the course of these patients' hospital stays. Central venous catheters with subcutaneous tunnels (e.g., Broviac, Hickman, Groshong) can stay in place for months. These tunneled lines can also be used for monitoring of cardiovascular status and are typically more comfortable for the patient. However, in the field of burn surgery, where managing patients with large often colonized burn wounds is commonplace, it is considered unconventional to place a tunneled CVC, especially when the tunneled CVC must be placed in proximity to the burn wound. Moreover, tunneled lines are generally not considered as frequently for central venous access in pediatric burn patients since placement of these lines are generally more invasive and costlier. As a result of this decreased favorability, placement of central lines with subcutaneous tunnel have been less studied in the pediatric burn patient population. Many studies have evaluated the use of central lines with subcutaneous tunnel in pediatric patients with hematological malignancies since these patients often require long-term venous access for administration of antineoplastic drugs, ${ }^{3,4}$ but no studies to date have specifically evaluated the use of tunneled central venous catheterization in pediatric burn patients.

At the Timothy J. Harnar Burn Center, which is an American Burn Association certified pediatric and adult burn center at the University Medical Center Hospital in Lubbock, Texas, pediatric burn patients sometimes receive tunneled central venous catheters when longterm venous access is likely to be necessary and PICC line placement is not a viable option. The number of pediatric burn patients who require central venous catheterization is relatively low, and only a small fraction of these patients require long-term catheterization. As a result of the small sample size and lack of any published literature on this topic, we present our findings here in a case series format. The lack of published literature on this topic highlights the need for more research pertaining to catheterization practices in pediatric burn patients. It is hoped that this case series will lead to more investigation into this topic and ultimately benefit the general population.

\section{METHODS}

With help from the Clinical Research Institute at Texas Tech University Health Sciences Center (TTUHSC), an application for this project was submitted, reviewed, and approved by the TTUHSC Lubbock/Odessa Institutional Review Board. To identify the applicable records to review, a report providing patient medical record numbers for specific individuals who met the inclusion criteria for this study was requested from the University Medical Center (UMC) Information Technology and trauma departments.

\section{Inclusion Criteria:}

\section{Patient age $<15$ years old}

2. Admitted to UMC between January 1, 2007November 1, 2017, with a burn injury

\section{Received a tunneled central venous catheter}

Using the electronic health records of the qualified patients identified in the report described above, we 
tracked the incidence of tunneled CVC-related complications. Two of the primary complications that accompany the presence of a CVC, regardless of approach, are catheter-associated infection and issues related to hemodynamics. Therefore, data concerning these complications were collected and presented in the case narratives presented below. Central venous cathetersassociated bloodstream infection was defined as a positive blood culture while the tunneled CVC was in place with concomitant clinical suspicion of CVC association. Issues related to hemodynamics included thrombophlebitis and embolic phenomena. Additional data that were collected for each applicable patient includes admission date, length of overall hospital stay, mechanism of injury, total body surface area (TBSA) of burn, central line dwell time and general demographic information.

\section{Patient naRRatives}

Patient one is a five-month-old female patient who had a $31 \%$ TBSA scald burn, affecting the buttocks and bilateral lower extremities. She was hospitalized for a total of 93 days. During her hospital stay she had eight surgeries, mostly for excision and grafting. A Broviac tunneled CVC was placed in the right internal jugular vein on hospital day two. The dwell time of this first tunneled CVC was 28 days (Table 1). Blood cultures positive for Enterococcus fecalis, Staphylococcus epidermidis, and Candida albicans were obtained while this first tunneled catheter was in place. A wound culture with the same bacterial species listed above was associated with this patient's first graft and ultimately led to graft rejection which necessitated removal and replacement. An infectious disease consultation was requested, and the clinical impression was that the bloodstream infection was not catheter-associated, but rather secondary to wound infection. No hemodynamic related complications were noted with this line. A replacement right internal jugular Broviac was placed on hospital day 28. Blood cultures positive for Candida albicans were obtained while this second catheter was in place. The clinical impression was that this bloodstream infection was also not catheter-associated. A major CVC-associated thrombotic event was detected via echocardiography 22 days after the replacement right internal jugular Broviac was placed which culminated in surgical thrombectomy of a blood clot which was located at the junction between the superior vena cava and right atrium. This patient was discharged 93 days following her admission with no other significant complications.

Patient two is a one-year-old male patient who had a $62 \%$ TBSA flame burn affecting his left upper extremity, left lower extremity, back, abdomen, and chest. He was hospitalized for a total of 90 days. He had 19 surgeries, mostly for excision and grafting. A Broviac tunneled CVC was inserted into this

Table 1. Catheter characteristics

\begin{tabular}{|l|l|l|l|l|}
\hline Patient ID & Tunneled CVC Characteristics & CVC Dwell Time & C-AT & C-AI \\
\hline 1 & Right IJV Broviac & 28 Days & No & No \\
\hline 1 & Right IJV Broviac & NA & Yes & No \\
\hline 2 & Right Subclavian Broviac & 29 Days & No & No \\
\hline 2 & Right Subclavian Broviac & 14 Days & No & Yes \\
\hline 3 & Right Femoral Broviac & 11 Days & No & No \\
\hline 4 & Right IJV Broviac & 48 Days & No & No \\
\hline 4 & Left Subclavian Groshong & 45 Days & No & No \\
\hline 5 & Left Subclavian Broviac & 27 Days & No & No \\
\hline
\end{tabular}

The average dwell time of the tunneled CVCs in this series was 28 days, and our analysis of the data revealed one tunneled catheterrelated infection and one hemodynamic complication.

C-AT-catheter-associated thrombosis; C-AI-catheter-associated infection; NA-no definite end of dwell per records in patient one's second tunneled CVC. 


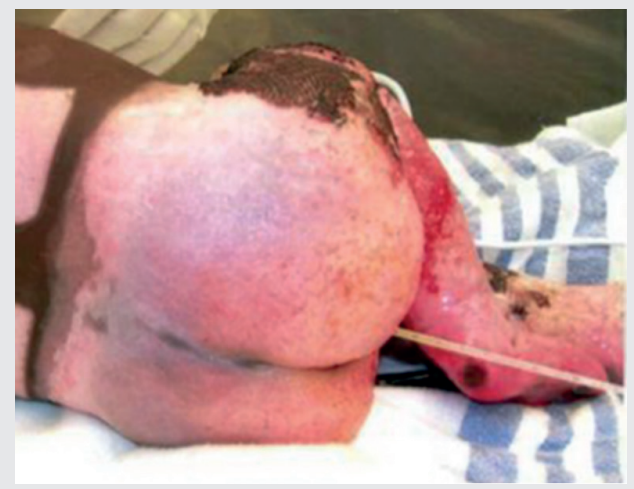

Figure 1. This photo demonstrates the extent of patient one's burn injury. $31 \%$ TBSA scald burn with significant third-degree involvement, affecting the buttocks and bilateral lower extremities.

patient's right subclavian vein. The dwell time of this catheter was 29 days (Table 1). This catheter was ultimately discontinued because of leakage. No positive blood cultures were obtained while this catheter was in place, and no hemodynamic related complications were noted. A replacement Broviac tunneled CVC was inserted into this patient's right subclavian vein following removal of the previous Broviac. The dwell time of this catheter was 14 days (Table 1). A blood culture positive for Serratia marcesans was obtained while this second catheter was in place and was believed to be catheter-associated. The infection was successfully treated with ceftriaxone. No hemodynamic related complications were noted while this line was in place. The patient was discharged on hospital day 90 with no other significant complications.

Patient three is a one-year-old male patient that had a $20 \%$ TBSA scald burn affecting his face, neck, torso, and left arm. He was hospitalized for a total of 14 days. He had one surgery for excision and grafting. Attempts were made at placing a PICC line in this patient, but they were unsuccessful. The burns on this patient's neck and chest made internal jugular veins an unfeasible option for central venous access. As a result of this, a Broviac tunneled CVC with subcutaneous tunnel on the anterior abdominal wall was inserted into the right femoral vein on this patient's fourth hospital day. The dwell time of the tunneled central venous catheter was 11 days (Table 1). No positive blood cultures were obtained while this catheter was in place, and no hemodynamic related complications were noted. The patient was discharged on hospital day 14 with no other significant complications.

Patient four is an 8-year-old female patient who had a $37 \%$ TBSA thermal burn, all third degree affecting her bilateral face, trunk, back, upper extremities, and lower extremities. She was hospitalized for a total of 79 days. She had fourteen surgeries for excision and grafting. A double lumen Broviac tunneled central venous catheter was placed in the right internal jugular vein on her seventh hospital day. The dwell time of this catheter was 48 days (Table 1). No positive blood cultures were obtained while this catheter was in place. No hemodynamic related complications were noted; however, the line insertion site was erythematous on hospital days 30-33. This line was removed due to breakage and replaced with a double lumen Groshong tunneled central line in the patient's left subclavian vein on hospital day 55 for the continued administration of fluids. No positive blood cultures were obtained while the second catheter was in place and no hemodynamic related complications were noted. The patient was discharged from the hospital to an in-patient rehabilitation center without removal of the second tunneled CVC. This catheter was removed 22 days after discharge. The dwell time

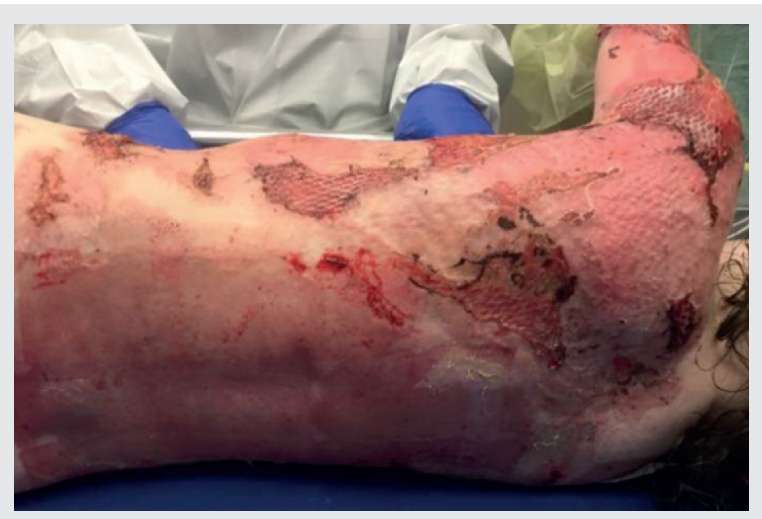

Figure 2. This photo demonstrates the extent of patient four's burn injury. 37\% TBSA thermal burn, all third degree affecting her bilateral face, trunk, back, upper extremities, and lower extremities. 


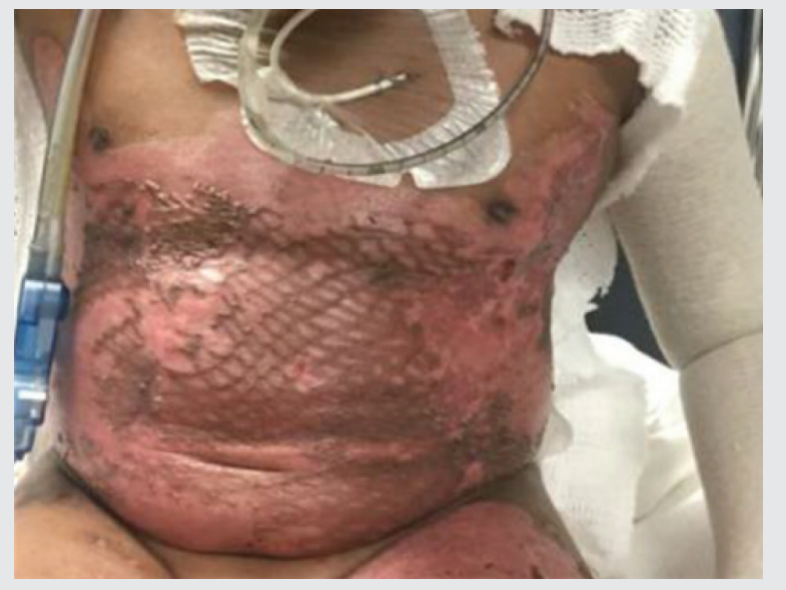

Figure 3. This photo demonstrates the extent of patient five's burn injury. $40 \%$ TBSA flame burn, $16.75 \%$ second degree, $23.25 \%$ third degree affecting his torso and bilateral upper and lower extremities.

of this second tunneled CVC totaled 45 days (Table 1 ). The patient was discharged on hospital day 79 with no other significant complications associated with either of the tunneled CVCs placed over the course of this patient's hospital stay.

Patient five is a 9-year-old male patient who had a $40 \%$ TBSA flame burn consisting of $16.75 \%$ second degree and $23.25 \%$ third degree affecting his torso and bilateral upper and lower extremities. He was hospitalized for a total of 47 days. He had five surgeries for excision and grafting. A single lumen Broviac tunneled CVC was placed in the left subclavian vein on his twentieth hospital day. The dwell time of this tunneled CVC was 27 days (Table 1). No positive blood cultures were obtained while this catheter was in place and no hemodynamic related complications were noted. The patient was discharged on hospital day 47 with no other significant complications associated with the tunneled CVC placed during this patient's hospital stay.

\section{Conclusions}

Among the five pediatric burn patients included in this case series, eight tunneled CVCs were placed.
Analysis of the data revealed one tunneled CVCrelated infection and one hemodynamic complication. One apparent benefit with using tunneled CVCs is the longer dwell time associated with these lines. The average dwell time of tunneled CVCs in this series was 28 days. Line discontinuations that were not related to infectious or hemodynamic complications in this series were for either subsequent surgical procedures in the region of the CVC, or CVC-related mechanical complications (e.g., breakage, leakage, etc.). Longer dwell times equate to fewer catheter insertions over the course of the patient's recovery. This is beneficial because acquisition of venous access in this patient population can be difficult, especially in pediatric burn patients with large TBSA involvement and in very young children. For example, if we look at the narrative for patient four above, we see that this patient had two tunneled CVCs over the course of 93 days. If following MAGIC guidelines, a 93-day venous access time course would require six non-tunneled CVCs. A study comparing cost and complication risk between tunneled CVCs and non-tunneled CVCs in pediatric burn patients could help to further elucidate the optimal method for venous access in this patient population.

Many healthcare providers believe that nontunneled CVCs and PICC lines are better to use with this patient population because they can be placed at the bedside, they are inexpensive, and they have better passive flowrates. The shorter dwell time associated with non-tunneled CVCs should be taken into consideration when providing care to pediatric burn patients where long term central venous access is likely to be necessary. Peripherally inserted central catheters are beneficial because they can remain in place for an extended period and because they can be placed at the bedside. However, PICC lines can be difficult to place in pediatric burn patients with large TBSA involvement. When PICC lines are not a viable option, tunneled CVCs appear to be a safe alternative since our data show a relatively low complication rate associated with the placement of long-term tunneled CVCs. Our small sample size warrants more investigation on this topic. A treatment algorithm is needed to identify good candidates for tunneled central lines based on injury characteristics 


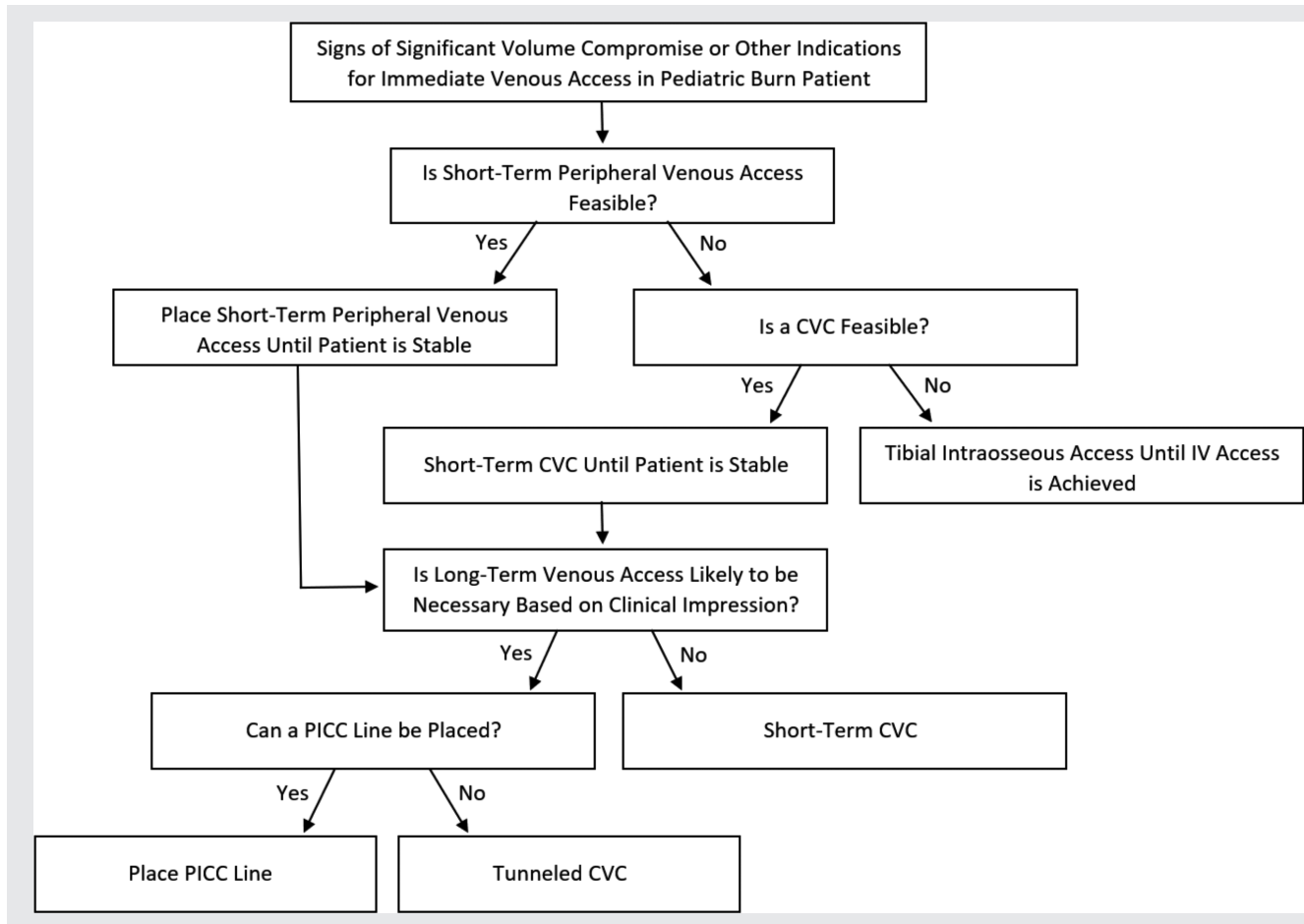

Figure 4. Treatment algorithm for pediatric burn patients requiring intravenous access.
(Figure 4). This appears to be an appropriate next step for this area of clinical investigation.

Article citation: Wagner C, Hess A, Olascoaga J, Van Spronsen N, Griswold J. Tunneled central venous catheterization, a viable option for long-term venous access in pediatric burn patients. The Southwest Respiratory and Critical Care Chronicles 2020;8(33): 29-34

From: Department of Surgery, Texas Tech University Health Sciences Center, Lubbock, Texas

Submitted: $10 / 28 / 2019$

Accepted: $12 / 27 / 2019$

Reviewer: Tetyana Vasylyeva MD, PhD

Conflicts of interest: none

This work is licensed under a Creative Commons

Attribution-ShareAlike 4.0 International License.

\section{REFERENCES}

1. Mattox K, Moore E, Feliciano D. 2013, Trauma 7th Edition. New York, NY: McGraw-Hill.

2. Chopra V, Flanders SA, Saint S, et al. The Michigan appropriateness guide for intravenous catheters (magic): results from a multispecialty panel using the RAND/UCLA Appropriateness Method. Annals Intern Med 2015;163:1-40.

3. Bergmann K, Hasle H, Asdahl P, et al. Central venous catheters and bloodstream infection during induction therapy in children with acute lymphoblastic leukemia. J Pediatric Hematology/Oncology 2016;38:82-87.

4. Cecinati V, Brescia L, Tagliaferri L, et al. Catheter-related infections in pediatric patients with cancer. European J Clin Micro Infect Dis 2012;31:2869-2877. 